E.B. virus, like other viruses, sometimes does produce lymphomas in animals. I would not be frightened by the prospect of developing infectious mononucleosis after E.B. virus inoculation and I would discount the danger of developing a Burkitt tumour in the short span of life left to me. The question I would regard as of the utmost importance to me is whether, by E.B. virus inoculation, my tumour could be made once more drugsensitive. The answer to this question is unknown but could, like many another scientific problem, be elucidated by being put to the test.-I am, etc.,

Department of Histopathology. R. SALM Royal Cornwall Hospital (Treliske) Truro

\section{Fingernail Findings}

SIR,-I read with interest the paper by $\mathrm{Dr}$. J. C. Robertson and Mr. M. L. Braune (2 November, p. 279). However, I consider that a few comments are called for.

It is well known that splinter haemorrhages of the fingernails are a common physical sign seen in normal healthy individuals and their most common cause is trauma. However, the fact that such haemorrhages are seen in normal persons does not detract from their importance in subacute bacterial endocarditis or other diseases, provided of course that they are not considered in isolation. The authors suggest that the distribution of splinter haemorrhages is statistically independent of that of pits. It seems misleading to employ statistical methods to compare two unrelated signs. Transverse splitting of the free edge of the fingernail plate is usually traumatic and has no disease associations.

It seems clear from the description of a young woman with Raynaud's phenomenon who showed lesions "like splinter haemorrhages" over the posterior nail folds that these were linear telangiectases. These are seen frequently in patients with Raynaud's disease and phenomenon, but may also be seen in other patients with cold extremities and in lupus erythematosus, dermatomyositis, and systemic sclerosis.-I am, etc.,

University Department of Dermatology,

Liverpool Royal Infirmary

Liverpool

\section{Facial Flushing in Acute Pancreatitis}

SIR,-Facial flushing in the absence of pyrexia is a frequently observed clinical sign in the initial 48 hours after the onset of acute pancreatitis. This sign has been recorded in $38(44 \%)$ out of 86 patients admitted with this condition. The flushing was readily observed in the initial 12 hours after admission in most patients, but a minority developed the striking appearance between 12 and 36 hours after admission. It is usual for the flushing to be present for approximately a day, but the "beacon-like appearance" which some of these patients exhibit lasts a much shorter time.

The presence of flushing is unrelated to the sex or age of the patient or the aetiology of the acute pancreatitis. It has little prognostic significance but can be a helpful diagnostic aid. Seventeen male and 21 female patients exhibited this sign and in four patients the disease proved fatal. This $10.5 \%$ mortality rate is similar to the overall mortality rate for acute pancreatitis reported from this hospital. ${ }^{1}$ It is interesting to note, with regard to the usefulness of this sign as a diagnostic aid, that two patients have been correctly suggested to have acute pancreatitis by a surgical ward sister on the basis of pronounced flushing in apyrexial patients with upper abdominal pain and vomiting.

The aetiology of the facial flushing may be related to the release of vasoactive peptides into the peripheral blood as a result of the pancreatic inflammation.-I am, etc.,

Department of General Surgery,

C. W. IMRIE Royal Infirmary, Glasgow 1 Imrie, C. W., Whyte, A. S., and Blumgart, L. H.,
Gut, 1974, 15, 340.

\section{Hiatus Hernia and Competence of Gastro-oesophageal Sphincter}

SIR,-In recent years there has been an undoubted swing of opinion towards attaching more importance to the inferior oesophageal sphincter itself in preventing reflux and less to the effect of ambient pressure outside it. This was largely due to a paper by Cohen and Harris ${ }^{1}$ that appeared three years ago and of which the conclusions seemed to have been accepted in leading articles in the B.M.f. ${ }^{2}$ and other journals. The authors claimed to show that hiatus hernia made no difference to compression in the inferior oesophageal sphincter or to the incidence of heartburn. However, the results were inconsistent not only with those of others but also with established principles of fluid mechanics, of statistics, or even of ordinary arithmetic. Moreover, the conclusions did not follow logically from the observations reported. An example follows.

What really matters in the sphincteric segment is, of course, total pressure-that is, inferior oesophageal sphincter muscle compression plus ambient pressure outside the segment; for it is this that must be exceeded by pressure in the gastric contents for reflux to occur. However, Cohen and Harris decided to determine only sphincter muscle compression. They tried to calculate this by subtracting simultaneously measured gastric pressure from total pressure in the sphincter. This gave them a near enough figure in normal people; but in the presence of hiatus hernia ambient pressure around the sphincter is intrathoracic pressure. By subtracting instead the higher gastric pressure, they arrived at much too low a figure for sphincter pressure in all hiatus hernia cases. Nevertheless, they claimed to have observed almost identical results in a group of 38 such patients. This could only imply that, in their hands, whenever a hiatus hernia was present sphincter muscle compression increased just sufficiently to compensate for the error in their method of calculation at the particular diameter of measuring apparatus that happened to have been used. By Laplace's law, for any given tension in a circular muscle the pressure produced within it is inversely proportional to its diameter. Thus at any other diameter some different tension would have been required to make their correction.

Many other mathematically extraordinary features, too numerous to describe here, will be enumerated elsewhere. ${ }^{3}$ These are also available to anyone who cares to write for them.-I am, etc., Royal Postgraduate Medical School,
London W.12

H. Daintree Johnson

1 Cohen, S., and Harris, L. D., New England Fournal of Medicine, 1971, 284, 1053.
British Medical fournal, 1971, 3,205.

3 Johnson, H. D., in The Oesophagus (in preparation). William Heinemann Medical Books Ltd. London, 1975.

\section{Screening for Sickle-cell Disease}

SIR,-Dr. D. I. K. Evans (19 October, p. 162) underestimates the dangers of the heterozygous sickle-cell trait. Apart from the recognized risks of flying in unpressurized aircraft or of anaesthesia with inadvertant anoxia there is another risk, that of swimming under water. Some 15 years ago I performed a coroner's necropsy on a man who died in a swimming bath. He swam 30 metres, under water happily, got out, and tried to repeat the process but died half way along the bath. The only abnormality at necropsy was the presence of sickle-cell trait and, from personal experience of the anoxia induced by trying this exercise, I am convinced that the trait was the cause of his death. In spite of the difficulties of the explanation, some words of warning really should be given to the carriers of this almost harmless trait.-I am, etc.,

Department of Pathology,

J. R. B. WILLIAMS Sister Hospital.

\section{Zuckerman Report and Radiology}

SIR,-Six years ago a committee was set up under the chairmanship of Sir Solly Zuckerman to consider scientific advisory machinery. After a long period of gestation in the Department of Health and Social Security, the recommendations of this Committee finally saw the light of day in April 1974 in the shape of a document (H.S.C. (IS) 16), which laid down certain organizational arrangements for the establishment of such machinery. They bear little relationship to the original recommendations.

We in Wessex believe this machinery to be unworkable. Not only is it top-heavy; it is largely composed of people who have no connexion with patient-handling. It appears, moreover, to be directed more at issuing instructions than at receiving advice from the sharp end of the service. Because of this the radiologists of the Wessex Region are of the opinion that no useful purpose will result from being associated with the scientific advisory machinery as presently constituted. We have therefore set up a regional radiological committee independent of the scientific advisory committee. We have explained to the regional health authority and to the regional medical officer in person our reasons for taking this action.

We are anxious that this should not result in any division between the radiologists and the radiographers, with whom there is complete sympathy and understanding. With this in mind joint meetings have been held between the radiologists and radiographers to try and establish some means whereby we can work in close concert. 